\title{
Effect of Average Conformity on Contact Stresses in Total Knee Replacement: Finite Element Analysis
}

\author{
Usman $^{1}$ and Shyh Chour Huang ${ }^{2}$ \\ ${ }^{1,2}$ National Kaohsiung University of Applied Sciences, Mechanical Engineering Department, 80778 Kaohsiung, Taiwan, R.O.C.
}

\begin{abstract}
The excessive contact stresses have been proved by many investigations for their role in the tibial insert failure. One parameter in the total knee replacement (TKR) design that influences excessive contact stresses is the average conformity. Thus, the aim of this study was to investigate the effect of the average conformity on the contact stresses in the TKR tibial insert. Ten three dimensional-finite element models with different conformity level were created. The conformity values of $0,0.2,0.4,0.6,0.7,0.8,0.9,0.95,0.99$, and 1 were applied to the sagittal and frontal plane of the TKR models. The results showed that the contact pressure and the von Mises stress were decreased if the average conformity were increased, and vice versa. And, the average conformity has a larger effect on the contact pressure than on the von Mises stress. Also, from these simulations, we were informed that the developing of the contact pressure and the von Mises stress in the TKR tibial insert was greatly influenced by the average conformity. And, the von Mises stress and the contact pressure had the same trend in response to the average conformity.
\end{abstract}

\section{Introduction}

The previous reports show that there is a positive correlation between wear intensity and contact stress level. The wear intensity is greater in low conformity than in high conformity total knee replacement (TKR) design [1]. The conformity is defined as the difference of radius between two articular profiles involved in a contact [2]. The conformity is attained by dividing the femoral component radius by tibial insert profile radius, to be between 0 and 1 . Where, 0 is the minimum conformity and 1 is the maximum conformity [3].

Although some other investigations reveal that the decreasing conformity can lead to lower wear, the low conformity may produce the other failure mode of TKR due to the excessive contact pressure [3]. Therefore, the conformity is still important to study in TKR design. Thus, the aim of this study is to investigate the effect of TKR conformity on contact stresses. In this study, the conformity value was represented by the average conformity of frontal and sagittal conformity.

Ten three dimensional-contact models with different conformity value, both in frontal and sagittal plane, were created in Solidworks and then simulated statically in Abaqus software. Due to the purpose of this simulation was to study the conformity effect phenomenon in TKR, the contact models of TKR were simplified by only using two parts: a curved indenter as the model for femoral component and a plane with dish-out-path for the tibial insert. The contact pressure and von Mises stress were then extracted from output database for analysis.

\section{Finite element analysis}

\subsection{Models design}

Ten three dimensional-finite element models with different conformity level were created. The sagittal (RFS) and frontal (RFF) profile radius of femoral component were kept constant at 37 and $23 \mathrm{~mm}$ [4], respectively (Figure. 1a). Instead, the sagittal (RTS) and frontal (RTF) profile radius of tibial insert dish (Figure. $1 \mathrm{~b}$ ) were varied to create the conformity level between femoral component and tibial insert dish. The conformity values of $0,0.2,0.4,0.6,0.7,0.8,0.9,0.95,0.99$ [5], and 1 , for both in sagittal and frontal plane of TKR, were chosen. The conformity values were then randomized by using Microsoft Excel to give all frontal and sagittal conformities the equal chance to pair [6], as we can see in Table 1 (column two and three).

By using the conformity values, the variation of profile radius of tibial insert dish, in sagittal and frontal plane, can be determined by dividing the femoral profile radius (RFS or RFF) with the corresponding conformity value. For example, model 1, RTF was obtained by dividing RFF (23 $\mathrm{mm}$ ) by the conformity in frontal plane $(0.7)$, it equals to $32.9 \mathrm{~mm}$. And, RTS was obtained by dividing RFS (37 $\mathrm{mm}$ ) by sagittal plane conformity (0), it 
equals to infinity. The same way was used for the rest of
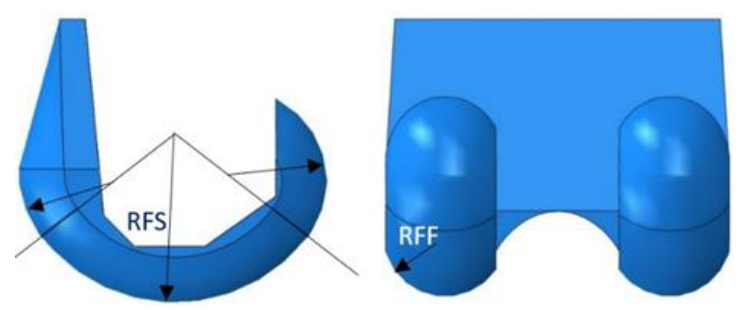

a) models.

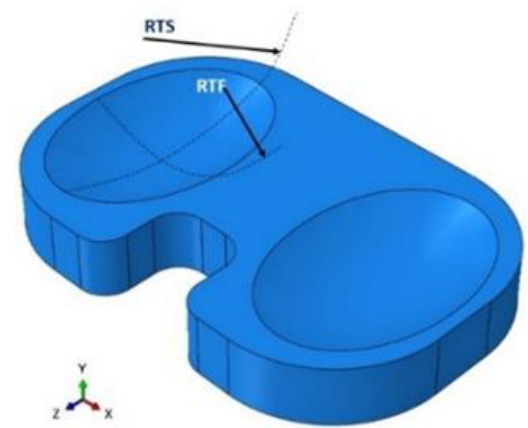

b)

Figure 1. a) RFS and RFF are the profile radius of femoral component in sagittal and frontal plane, respectively; b) RTS and RTF are the profile radius of tibial insert dish in sagittal and frontal plane, respectively.

Table 1. The conformity and measurement of tibial insert dish both in sagittal and frontal plane.

\begin{tabular}{cccccc}
\hline \multirow{2}{*}{ Model No. } & \multicolumn{2}{c}{ Conformity plane } & RTS $[\mathrm{mm}]$ & RTF $[\mathrm{mm}]$ & Average conformity \\
\cline { 2 - 3 } & Frontal & Sagittal & & 0.9 & 0.7 \\
\hline 1 & 0.7 & 0 & $\sim$ & 32.9 & 0.8 \\
2 & 0.9 & 0.7 & 52.9 & 25.6 & 0.875 \\
3 & 0.95 & 0.8 & 46.3 & 24.2 & 0.8 \\
4 & 0.6 & 1 & 37.0 & 38.3 & 0.7 \\
5 & 1 & 0.4 & 92.5 & 23.0 & 0.945 \\
6 & 0.99 & 0.9 & 41.1 & 23.2 & 0.2 \\
7 & 0.2 & 0.2 & 185.0 & 115.0 & 0.6 \\
8 & 0 & 0.6 & 61.7 & $\sim$ & 0.675 \\
9 & 0.4 & 0.95 & 38.9 & 57.5 & 0.895 \\
10 & 0.8 & 0.99 & 37.4 & 28.8 & \\
\hline
\end{tabular}

The models were simplified by only using the medial part of TKR (Figure. 2) due to symmetric TKR design. This is because of the medial part supports larger portion of knee joint load than lateral part at $60-40 \%$ ratio as also used by Halloran et. al. [7]. The femoral component was represented by curved shell with sagittal and frontal profile radius of 37 and $23 \mathrm{~mm}$, respectively. And the tibial insert was represented by a plane containing a dish with sagittal and frontal profile radius as tabulated in Table 1 (column four and five), respectively. This way of simplifying model followed the work of Wang et. al [8]. The minimum thickness of tibial insert of $8 \mathrm{~mm}$ was maintained to follow Bartel et al.'s recommendation for the minimum thickness of tibial insert [9].

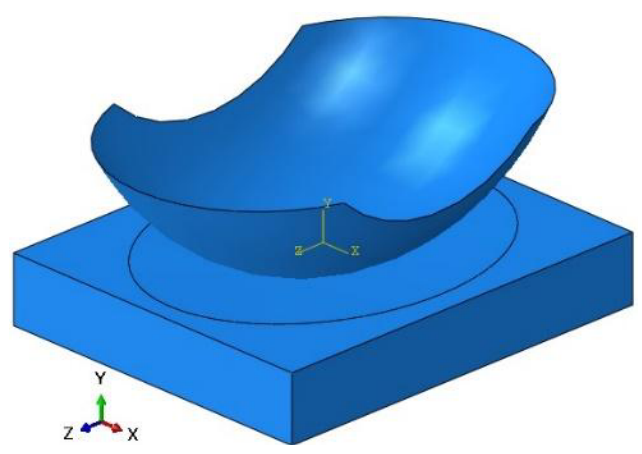

Figure 2. The simplified of TKR contact model for finite element analysis.

\subsection{Material properties}

The tibial insert was modeled as a deformable body with UHMWPE as its material ( $\mathrm{E}=550 \mathrm{MPa}$ [7], $v=0.44$ [3]). Meanwhile, a rigid body assumption was applied to the femoral component because the $\mathrm{CoCr}$ (the material of femoral component) modulus of elasticity is much higher than UHMWPE. The nonlinear true stress versus true strain and coefficient of friction of UHMWPE were used as in the previous work of Halloran et. al. [7].

\subsection{Loads and boundary conditions}

The vertical knee joint load during descending stair of $400 \%$ bodyweight [10] was applied at the femoral component reference point. The bodyweight of $700 \mathrm{~N}$ was chosen. Due to the simulation was only in medial part, $60 \%$ of load portion was used. Thus, the total vertical load equaled $1680 \mathrm{~N}(400 \%$ x $60 \%$ x 700$)$. The femoral component was only allowed to translate in vertical direction, while the tibial insert was fixed in all directions.

\subsection{Finite element Models.}

The finite element analysis was conducted in ABAQUS software. The Solidworks was used for creating the 
models and then inputted into ABAQUS. A discrete rigid element (R3D4: 4-node 3-D bilinear rigid quadrilateral) with $0.75 \mathrm{~mm}$ of global mesh size was used for femoral component. And the tibial insert was discretized by using 8-node linear brick element (C3D8) with $1 \mathrm{~mm}$ of global mesh size. The finite element is shown in Figure. 3.

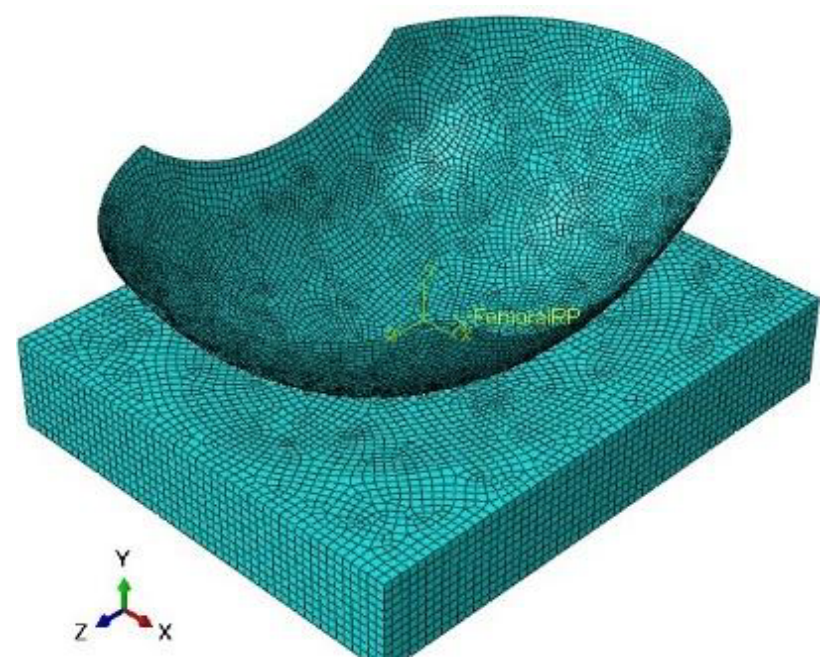

Figure 3. The finite element model.

\subsection{Results}

The Figure 4 shows the contact pressure (left) and von Mises stress (right) for all models. From the figure, we can see that the contact stresses have the same trend with the von Mises stresses. As also can be seen in the figure, the von Mises stress will increase if the contact pressure increases, and vice versa. The smallest contact pressure and von Mises stress of 11.44 and $9.62 \mathrm{MPa}$, respectively, were occurred in the model 6 with the average conformity of 0.945 . By this result, we got the evidence that the average conformity has a great influence in developing contact pressure and von Mises stress in the TKR tibial insert. The largest contact pressure and von Mises stress were occurred in model 7. This model has the lowest average conformity of 0.2 . Therefore, the contact pressure and von Mises stress increased to 37.48 and 20.46 MPa, respectively.

The average conformity was a new parameter we introduced in TKR design. Compared with the only conformity which merely represents the conformity in one plane (frontal or sagittal) of TKR, the average conformity is more important to study because it represents the conformity in both frontal and sagittal plane at the same time.

Through this study, we propose the average conformity as a parameter that should be considered in TKR design in the future. This is because, to the authors' knowledge, the average conformity has never been used in TKR design.

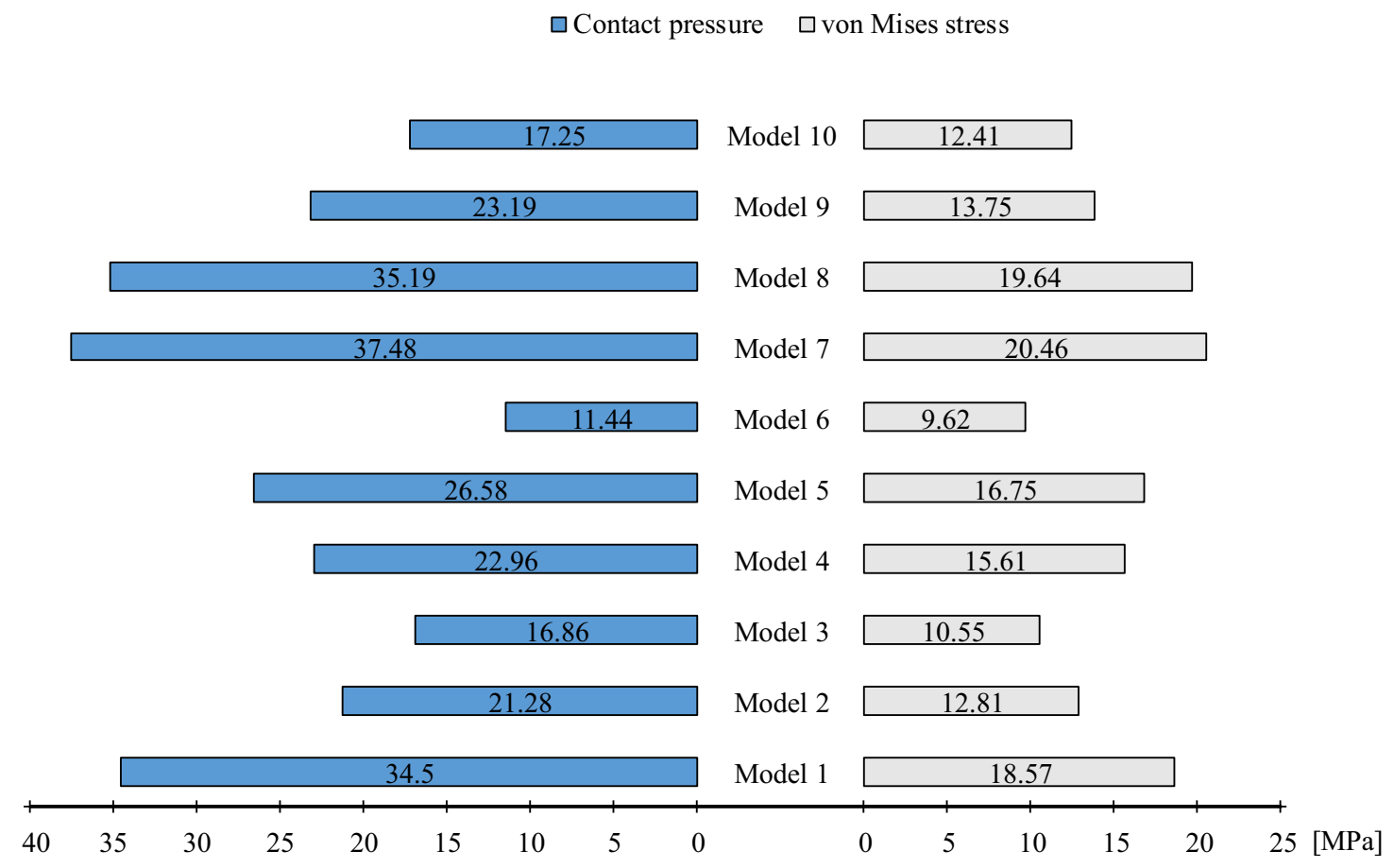

Figure 4. Contact pressure and von Mises stress in all model.

By using the linear regression (Figure. 5) we can see that the contact pressure (CP) and the von Mises Stress (VMS) decreased by the increasing of average conformity (AC). It also showed that $93.9 \%$ of contact pressure in tibial insert was explained by the average conformity, while the von Mises stress (VMS) was at $85.75 \%$. From the gradient of the regression line, we know that the contact pressure was more sensitive to the average conformity than the von Mises stress. For the contact pressure, the gradient was - 31.152, while 12.952 was for the von Mises stress.

In the figure, we found that the data are only concentrated around the tip of regression line. This is because the conformity values used in this simulation 
were taken from the previous literature where the conformity is selected from the rational value of the conformity in TKR. So, the data did not spread along the regression line. However, with the limited conformity

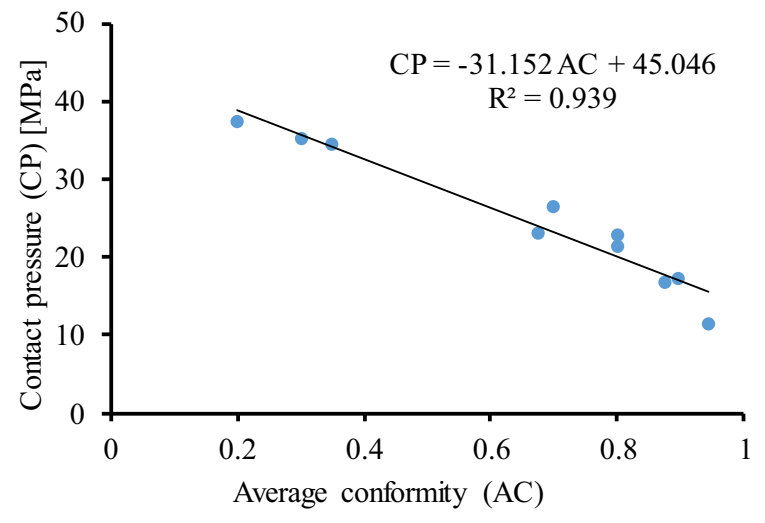

a) data presented in the literature, it is enough to be used to know the average conformity effect on the contact stresses as shown in Figure 5.

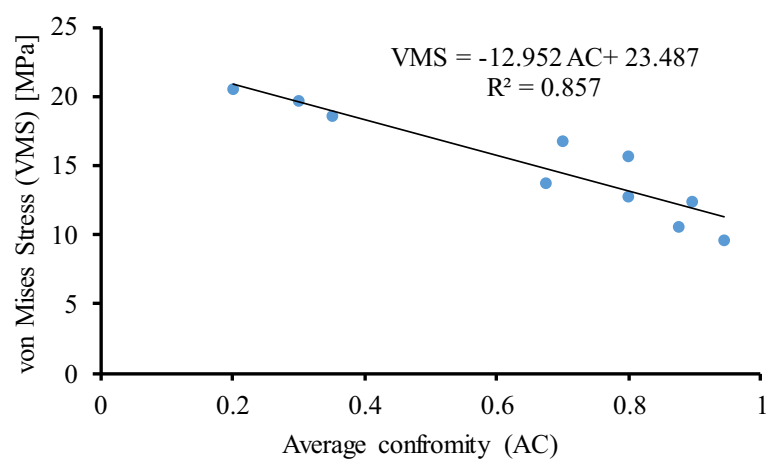

b)

Figure. 5. a) The relationship between average conformity and the contact pressure in tibial insert of total knee replacement. Meanwhile b) is the relation of average conformity with von Mises stress.

\section{Conclusions}

We have conducted the finite element simulation of ten models with different value of average conformity. From the result, we found that the contact pressure and the von Mises stress were decreased by the increasing of the average conformity, and vice versa. Compared with the von Mises stress, the contact pressure was more sensitive to the average conformity. Along with these simulations, we also got the clarification that the developing of contact pressure and von Mises stress in the TKR tibial insert was greatly influenced by the average conformity. And, the von Mises stress and the contact pressure had the same trend in response to the average conformity. Based on its effect on developing contact stresses in the TKR and its representation of sagittal and frontal conformity, the average conformity should be considered as design parameter in TKR design in the future. To the authors' knowledge, the average conformity has never been used in TKR design.

\section{Acknowledgment}

We would like to show our gratitude to the Minister of Sciences and Technology of the Republic of China for their partial financing this study under Contract Number MOST 105-2221-E-151-016.

\section{References}

1. J. P. Collier, M. B. Mayor, J. L. McNamara, V. A. Surprenant, R. E. Jensen, Clin. Orthop. Relat. Res. 273, 232-242(1991).

2. M. M. Ardestani, M. Moazen, Z. Jin, Knee 22, $217-$ 224 (2015).

3. R. Willing, I. Y. Kim, Struct. Multidiscipl. Optim. 38, 105-414 (2009).
4. H. C. Jhao, J. Y. Wang, Construction of Custommade Artificial Knee Joint by Means of Contact Information (Master Thesis in Institute of Electrical and Mechanical Engineering, Cheng Shiu University, Taiwan, 2013).

5. M.S. Kuster, S. Horz, E. Spalinger, G.W. Stachowiak, A. Gächter, Clin. Orthop. Relat. Res. 375, 302-312 (2000).

6. D. S. Moore, G. P. McCabe, B. A. Craig, Introduction to the Practice of Statistic, sixth edition (W. H. Freeman and Company, United States of America, 2009).

7. J. P. Halloran, A. J. Petrella, P. J. Rullkoetter, J Biomech. 38, 323-331 (2005).

8. C. Y. Wang, H. C. Jhao, C. F. Huang, Life Sci J. 10, 254-258 (2013).

9. D. L. Bartel, V. L. Bicknell, T. M. Wright, J Bone Joint Surg Am. 68, 1041 -1051 (1986).

10. I. Kutzner, B. Heinlein, F. Graichen, A. Bender, A. Rohlmann, A. Halder, A. Beier, G. Bergmann, J Biomech. 43, 2164-2173 (2010). 